\title{
CONHECIMENTO E ESTEREÓTIPO DE TRABALHADORES ACERCA DA HIPERTENSÃO
}

Knowledge and stereotypes of workers concerning hypertension

Conocimiento y estereotipo de trabaj adores respecto a la hipertensión

MeriaEuridéa deCastro

Meysa Qivera Rolim

\section{Resumo}

Aprevenção dahipertensão ateria toma-sedifíl devidbàs dfia ldades deacesso aosistemadesaúde Foramobjètivos desteestudb: averiguar o corhedimento dos trabalhadores acercada pressão atterial, dahipertensãoe, ainda, a prática da verificação da pressão arterial. Trata-se de umestudo descritivo, realizadb emuma UniversidadePública

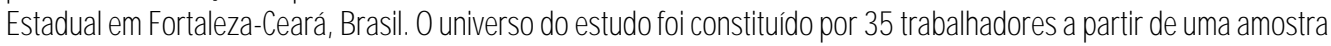
aleetóriasimples. Depreendeursequehádéficitdecorhedimento acerca dap pressão aterial edahipertensãoe equers questões alturais entre profissionais e usuários do sistemadesaúde estão emdesacordo como processo ensinoaprendzagem Conduiusequeurgemestrdégas quepermitam maior adesão aos programæs eàscamparhes dehipeten-

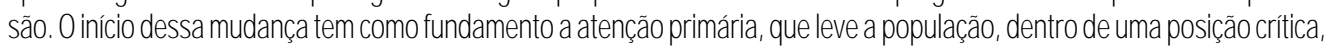
atomar atitudes quepossibilitemadequer-seaumacondição devicasandánel.

Palaraschave PressãoAtterial. Hpertensão. Effermagem

Abstract

Theprevertion of arterial hypertensionhes notbeenaneesy Laprevendóndelahipertensiónaterial es difíl por d acceso

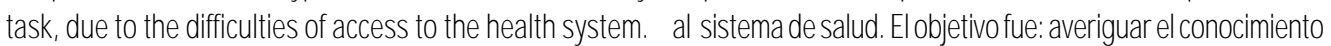
Thedgedtives of this studymereto ascertaintheanount of detrabajadoressobrelatensiónaterial ydelahipertensión norker's knowedge conceming blood pressure and y, adenás, la prádicadelaverificacóndelatensiónateria. hypertension as well as about the pradice of nesuring Setratadeunestudiodescriptio, realizadbenla Uhiversidad bloodpressure This descriptivestudymas doneattheState PúdicaenFortalez-CeráB Brasil. Fueronseleccionados 35 University in Fortaleza-Ceará The universe ves a simple indviduos. Seconduóquehaydéfictdeconocimientodela randomsample of 35 norkers. Alackof knowedgeabout tensiónaterial ydelahipertensión quelos planteamientos bloodpressureandhypetersionvesfound asvell asatturd atturdesentreprofesiondesy usuarios de sistermestánen issues between professionals andsystemusers notbeingin desaaverdocone proceso enseñanzz/aprendizaje Urgen agreamert with the teaching-leaming process. It weS estrdegiesquepemitanumamayor adhesiónal programa y condudedthatstrategieswichallowfor a wider partiaption comricación efectiva al nivel de entendimiento de las in theprogramandinhypettersion campaigns areneeded. persones. Einiciodeesecanbiotienecomofundamentola The begiming of this changehas as a basis primary care a atencón primaria, llevandb a la poblacón, dertro de una wichallousthepopulationtonakechaiceswichfitaheathy posicóncutica, tomaraditudesqueposibilitenadeararsea lifestyle, fromacritical position

\section{Keymords}

Bloodpressure Hypertension Nursing

\section{Palabasdace}

TensiónAterial. Hpetensión Erfermería 
INTRODUÇÃO

As pessoes experimentam diferentes sensações dante das alterações no estado de saúde, principalmente em casos de dbenças crônicas, como a hipertensão aterial sistêmica (HAS). Nessa erfermidade, as estruturas física e psicossodal dos indiv́duos são afetadas, necessitandb, então, que estes adbtemumestilo de vida saudável para nanter o equilibrio da pressão arterial.

A hipertensão é una doença multifatorial, de arso assintomático e prolongadb, que predisa de una assistênda à saúde contínua, e, geralmette, está associada a res trições e adbções de esqueras terapâticos complexos'.

É uma dbença de alta prevalência no Brasil, a qual atinge cerca de $20 \%$ da população adulta joveme $50 \%$ da população idbsa, contribuindo expressivemente para o grande número de óbitos anuaiš.

Nb Brasil, há umhipertenso para cada três pessoas comidade aaina de 40 anos $^{3}$, e, aproximadanente, $85 \%$ das pessoss que apresentaramadidente vesalar encefálico e $40 \%$ das vítinas de infato do miocárdo relataramHAS associada Dadbs do Instituto Nacional de Seguro Social (INSS) denenstramque 40\% das aposentadorias precoces decorremdesse tipo de enfermidadet.

Enbora a causa da hipertensão atterial seja descomecica, váios são os fatores que podemestar associa dbs à eleveccão da difra tensional, como o sedantarismo, o estresse, o tabagismo, o envelheaimento, a história familiar, a raça, o gênero, o peso e os fatores detéticos 5 .

O controle da HAS tomourse umdesafio para os profissionais de saúde e, embora os dados da Organização Mndial de Saúde (ONB) revelemque $45 \%$ dos profissionais de saúde emtodo o mundo não estão treinados para lidar coma hipertensão, hoje, são 600 milhões de hipertensos no mundo, dos quais $500 \mathrm{~m}$ Ihões predisam de intenvenção médica imediatá.

Atualmette, ênfase é dada na orientação de reddas não farmacológicas, proporcionandb a todos os hipertensos e às pessoas comforte antecedêndia fa miliar, opções para u ma mudança no estilo de vida.

É fundamental que o profissional de saúde, ao abordar um hipertenso, preocupe-se com as percepções do indivíduo quanto a sua doença, propiciando meios para o conhecimento da sua condição de saúde, e ao conseqüerte desenvivimento da auto-responsabilicadel.

Dante dsso, a Erfermagemassume inportânia funda

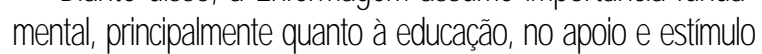

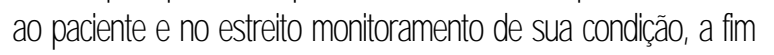
de promever nethorias ra sla situeção de sádedbençå.

Nbs úttimos anos, a educação à saúde vemse constituindo una preocupação para o enfermero, talvez por exigênias do próprio papel social da profissão, que estimila e requer una reaveliação dessa prática?.

Oenfermero deve considerar o contexto alturad do individuo ao tratar das questões inerentes ao processo saúdedbença, no que tange às causas, sintomas e eficá da de ara, estilo de vida. Dessa forma, urge que esses profissionais "se apaixonem" pelas pessoas e participem do seu mando. Assim, será possível una refleeão quanto ao preconceito de que os dientes nada sabemsobre as questões de saúde e dbença

Enbora haja maior interesse dos profissionais de Enfermagempelas ações educativas, a maioria ainda acredita que os conhedimentos e as informaçoes transmitidas são os principais fatores da educação.

Quato a isso, mo processo de ed caçã̃o popular, não é possivel aos educadores pensar somente nos procedmertos didáticos e nos conteúdos a seremensinados aos gupos populares, nas tambémque estes estejamcoe rentes coma realidade do gupo a ser educado ${ }^{10}$.

Preverir a hipertensão rão temsidb fáail, tanto para os indvíduos assistidbs pelo serviço de saúde quanto para os profissionais, emdecorrêndia das implicações db sisterm de saúde passadb, aja organização se baseau na política nédica e as questões de saúde eramde responsabilidade estritamente indvidual.

Adhourse oportuno desenvolver umestudo, que se originou a partir de una pesquisa realizada comtra balhadores de uma Universidade Pública, durante o qual se objeivou averiguar a prevenção e controle da pressão arterial comvistas à adoção de umestilo de vida saudável. No entanto, identificaramse setores com predomínio de pessoas hipertensas, destacando-se o Restaurante Universitánio, devido à característica pea liar do trabalho ali desenvolvido. Para tanto, e pela necessidade de interagir como gupo e incentiválo a participar de ações educativas, fez-se necessário investigar a noção que os trabalhadores tinhamacerca da hipertensão arterial e sues reperaussões no cotidiano. O trabalho é relevante por destacar a prática de nedicas de prevenção e controle que devemser adbtadas, entre elas a mensuração da pressão aterial.

Ovelores de pressão aterial e de hipettensão addtadbs neste etudo forama pressão ateria sistólica (PAS) $=12$ $\mathrm{mmth} 140 \mathrm{mmth}$ e a pressão arterial dastólica (PAD) $=80 \mathrm{mmtg} 90 \mathrm{mmtg}$ respectivenerte

Enbora exista una vesta literatura sobre o tema, a deficiente prevenção da hipertensão é responsável por mitos agravos à saúde da populaçãa. Assim, são dojejvos deste estudb: averiguer o corhedimto dos trabalha dores acerca da pressão aterial e da hipertensão e, ainca, verificar a pressão arterial rotineiramente 
MATERIALE MÉTODO

Oestudo é de natureza descritiva, comenfoque qualitativo, realizado no Campus de una universidade púdica estadual da cidade de Fortaleza-Ceará, no nếs de março de 2003.

Utilizourse, como instrumento de coleta de dados, umroteiro de entrevista, comperguntas norteadoras acerca da pressão arteria e da hipertensão, bemcomo da freqüênia comque verificama pressão sangünea, tendo sido aplicadb após umpréteste.

O universo do estudb constou dos trabalhadores do Restaurante Universitánio. Aamostra aleatónia sim ples computou 35 trabalhadores que responderamà entrevista durante a coleta de dadbs, os quais se encontravamno ambiente de trabalho no momento do estudo e aceitaram voluntariamente participar do ensaio após as informações prévias.

Os dados foramtranscritos, e fai realizada una leitur ra flutuante nas falas, que foramcategorizadas por similitude emuridades teráticas, conforme Bardin ${ }^{11}$, selecionadas de acordb coma proximidade dos objeilos , asaber. definição de pressão ateria; corheainerto sobre hipertensãa; mensuração da pressão sangün $e^{10}$.

Oprojeto foi aprovedo pelo Comitê de Ética emPes-

quisa da Universidade Estadual do Ceará (UECE), sob processo nP020663641, conformea Resolução 196/96, ${ }^{12}$ e, aos entrevistados, foramassegurados o sigilo das informações, o anorim to e o liverearbátrio na indusão do estudb. Ainda foraminformados sobre o Termo de Consertimento LiveEsdaredido, assinado pelo participante, logo após o término da entrevista

\section{ANÁLISE E INTERPRETAÇÃO DOS DADOS}

Caraderização sociodenrográfica entre os 35 ettre vistadbs, 23 (63\%) são do sexo nasalimo, 18 (51\%) pertencemà faixa etária de 40 anos ou mais, 16 (46\%) possuemo ensino fundamental incompleto, 23 (72\%) possuemrenda faniliar entre 3 e 7 saláios nínines, 14 (40\%) apresentamantecedêndia familiar para hipertensão e 15 (43\%) são portadores de hipettensão.

Quato à pressão aterial, 11 (31\%) entrevistados encontravemse entre o intendo de 132-190 mity para a pressão atterial sistólica e 90-110 mitty para a pressão arterial distólica, sendo que 4 (17\%) dos demais entrevistadbs, entbora a nédia da pressão terha sidb inferior a $140 \times 90 \mathrm{mmth}$ dimmamfzzer uso de redcação hipotensora Indivído coma sistólica 120 a 139 mittg e a dastálica 80 a 89 mittg deve ser considera do préhipertenso ${ }^{13}$ e requer pronoção da saúde.
As falas dos entrevistados resultaramnas seguintes categorias em destaque:

Definição da pressão arterial

A respeito dessa unidade terática, detectourse que 25 (71\%) dos trabalhadores desconhecemo significado de pressão arterial, associando sua ignorância à ausência de uma educação eficaz nas instituç̧ões de saúde Obsenvemse os depoimentos:

Nấo sei o que é não, não terho a menor idéa do queseja (E2, E3).

Éuna dbença que mata a pessca semaisar, ausga ...é conseqüênia de dbesidade, vida sedertária, excesso de sal, deálcod, tudb isso ocasiona a pressão atta, conø tantbémofator deheredtariedade (ET).

Falar eu oun, mas uma coisa que a pessoa fala, mas não explica, né? tá na parede pregada... mas rão se entende (E9).

Pressão arterial é a velocidade comque o sangue circula nas veias, a média é 10 × 8; uttrapassando, por exemplo a 14, é considerada alta É prejudidal à saúde, pode dar enfarto (E31).

Eu não sei nada sobre pressão aterial e mito me nos a dasse rédica porque hoje mato a respeito de saber a causa e ainda não consegui; segundo alguns rédicos que eu estive agora emSão Paulo ...disseramque até hoje ignoramredmerte a razão da pressão arterial (E32).

Apesar do reconhecimento do trabalho da enfermera, dos programas existentes, observourse que os ensinamertos e os nateriais didáticos dixados não servirampara esdarecer as questões sobre hipertensão.

Assim deve-se atentar para uma prática transformadora, aja preocupação dos profissionais é considerar as peauliaridades das pessoas, valorizando sua satisfação coma terapêtica, como autoaidado e comos mecanismos uilizados para que a informação seja transferida de forma simples e acessivel à popur lação-alvo, procurando respeitar os aspectos socioeconômicos e alturais.

Houne, também aqueles que responderamde forma impredisa e outros que confundirampressão ate rial, nas de certa forma corhecemalgo sobre prevenção e tratamento da hipertensão e até mesmo algut mas complicações, como se verifica emalgumas falas:

Pressão aterial é relacionado como sangue, né? Que o sangue passa nas artérias, né isso? ....se comer gordura, aí vai criando aquelas placas e im 
pede o sangue de se movimentar liverente, á dá assimumerfarto (E22, E28).

Pressão arterial é o sangue, o movimento do sangue, né? Eu acredito que seja isso. Coma idade vai se agravando mais, né? Dependendo tambémdo estilo de vida da pessoa (E18).

...Eusi quépragentedminir gordra o sa (E13). ...Nâo beber certas bedicas...(E13, E28).

...Fazer camirhada né? (E9, E6).

...Tonar os medicamentos na hora certa (E9).

Apesar de não teremconheaimento coerente sobre pressão, sabemque precisamtomar certos aildadbs e se subreterema alguns sacifíicos, tais com: exerácios físicos, restrição ao álcod, mudança na alimentação; haja vista trabalharemcoma manipulação de alimentos, mestro assimnão conseguemseguir as redidas preventivas.

Partindo de umestado de nommalidade, pressão arterial referese à pressão do sangue exercida sobre as paredes das artéizas ${ }^{14}$, sendo responsável pelo sur primeto de sangue emtodb o sistema human, mantendb o ser emestado de equilibrio ou emcondições de saúde desejáveis.

\section{Aprendizagem sobre hipertensão}

Quando indagados acerca do que tinhamaprenddo sobre hipertensão arterial, depreendeurse que 19 (54\%) trabalhadores têm concepções equivocadas, ainda que todos freqüentemos seniços de saúde, re cebamorientações e, mesmo assim assumamhaver esquedido do que se trata a HAS, conforme se observa nos depoimentos abaixo:

Sé não, (E1, E9, E31) já aù falar mito, mas não entendb (E9), não entendo a linguagem(E2).

É a doença que tá emtodo mundo, prinapalmente empessoa de mais idade ...agora o problema eu rão sei o queénão (E 18)

o meu a rhado estava bom de repente teve um enfarto, tinha feito todos os exares...(E 11).

Hpertensão é dor na nuca, né? É una pessca agitada denais, querer resolver as coisas depressa... (E19).

Tipo assimnaurose, né? Faz a pressão subir (E24). ... mitas coisas fazemela alterar, preocupação, alinentação, excesso de trabalho (E32).

Eu já li sobre hipertensão arterial ...deixa eu ver ...pessoa hipertensa ...eu li autro dia umbocado de artigo...eu não me lembro...eu gostaria que você me dissesse, que vai ser bom (E21).
Nấo sej, porque esse pessoal que tira a pressão da gente não explica nada, né? Faz tá tanto por tanto, pronto, aí escreve lá.. (E1).

Euaprend quepressão ateria éa velocidadecomqueo sanguedirala res vies, a nédaé $10 \times 8$, utrapessandb, por exmplo a 14, éconsidarada alta (B).

Pode-se obsenvar que, apesar das orientações prestadas pelos profissionais, da existência de Programa de Controle da Hipertensão e das camparhas realizadas, as pessoas ainda não estão esdarecidas.

Ressalta-se ainda o distanciamento da comnica ção técrica dos profissionais da saúde que não vem contemplando as démidas dessas pessoas, haja vista que não são capazes de compreender o que significa pressão alta, nemtampouco os riscos que ela pode ocasionar. Da mesma forma, o material didático afixado em locais espećficos não tem sido autoexplicativo.

A pronroção de saúde por meio de ações educatives pode originar auto-responsabilidade, adbção de um estilo de vida saudável, redução dos fatores de risco, bermcono dos sintomæs da doença crônica e melhoria da qualidade de vida?.

É visível a necessidade de ações educatives que possibilitemà população refletir e aprender. E a Enfernagem comseu papel educador, pode prestar assistêndia por mie da detecção precoce do risco cardiovesaular para diminuir a morbimortalidade por esse tipo de doença ${ }^{15}$.

Assim a prática educativa temrelevância no sentido de manter ou melhorar as condições de saúde dos trabalhadores, emcasos de agravos do sistena cirar latório, permitindo que estes tomemcertas atitudes emrelação ao seu estado, possibilitandb a adesão de umestilo de vida coerente comseu padrão de saúde.

Mensuração da pressão arterial

Qunto a essa categoria, 13 (37\%) trabalhadores proaramverificar a pressão atterial como medida para pre verir a hipertensão. Entretanto, ressaltamo não-esdare aimerto dos velores pressónicos pelos profissionais. $\mathrm{Ob}$ senverise as falas:

Tá mais que ummế.... doutor disse: "sua pressão tá ớtima, a pressão de ummenino". Tambémnão disato, né? (E4).

Esse negócio de pressão é umnegócio complexo, sabe por quê? Aonde eu moro temánco famácias, ...! Á sá cla primera đéa quinta Fiz exame da pressão. Cada uma deu diferente da outra, nenhuma 
deu igual! ...Se eu for hoje dá 13×8, amanhã dá 17x10 aí nesse negócio (E16).

Eu vejo a pressão comessas menininhas que ficampor á ...(E12)

Embora a aferição da pressão atterial através do nétodo indireto seja umprocedimerto simples e fádil, o dfíil é o acesso das pessoas aos programas de hipertensão institúdos pelo Mristéio da Saúde

Ormod de aveliar a pressão aterial é umfator im portante Por isso, an se mensurar a pressão arterial de umindivíduo deve se dar ênfase ao controle tanto do aparelho como do profissiond que o usa' ${ }^{16}$.

A hipertensão arterial linítrofe para o indvídu aduto, maior de 18 anos, são valores de pressão arterial sistólica entre 139 e 130 mmtg e pressão arterial dastólica entre 89 e $85 \mathrm{mmth}^{77}$.

Apesar de ser considerada hipertensão, una pressão arterial igual ou maior que 140/90 mHtg há os casos de pré hipertensão registrados nas pessoas om pressão de 120/80 mity. Esses não são considarados portadores de hipertensão, næs apresentammaior nisco cardiovesalar e estão mais propensos a desenviver hipertensão do que indvíduos comníveis pressónicos mais baixos se rão adbtarem umestilo de vica saudánd ${ }^{13}$.

\section{Referências}

1. ReisMGGashenRQ Adutoshipertensos hospitalizados: percepção degravidadedadbençae edequalidadedevida RevLatino-Am Enfermagem2001 maio; 9(3): 51-7.

2. Oivera TC,Araíjo, TL, MaloEMAlreica, DT.Adiaçãodoprocesso adaptativo de umidoso portador de hipertensãoarterial. Pev Latino-AmEnfernagem2002ju/ago; 10(4):530-36.

3. SociedadeBrasileiradeH Hettensão(BR). Consensolrasileirode hipetensão aterial. 1998, Campos dbJordăo(SP): SociedadeBra sileiradeH pertensão; 1998.

4. Mristéio da Saúde(BR). HpertensãoArterial Sistêmica(HAS)e Dabetes Mallitus (DM): protocolo. Braslia(DF); 2001.

5. MdiraMBB, CriraRS HelkehoflL,MIIJGHpetensãoaterideconst nodesal empoulaçãourbara PeVsádePídica2003; 37(6): 743-50.

6. BrandāoAP, NbgueiraAR KohlmamJuniorQ, Cavelha, MH GorgiD, Sartos Reta. Camparhaserámarcadapor evertos emdiversoslocalidades do pás. SociedadeBrasileiradeH Hpetter são. [online] 2004;

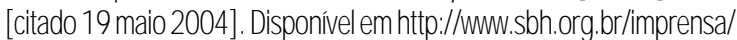
reese_SB__CRG_BR_Comparha\%/20Nbaiond_2004.pof.

7. Harbon EMedcdandconplentertary.NusTimes 2002jan98(2):32-4.

8. Coady R Cronicheartfailure NursTimes 2002ju;; 98(30): 41-4.

9. Mrticelli MAsaçõesed cotivesemeremagemdosensocomemao bomsenso. TetoscontetoErferm1994jivdez; 3(2): 7-15.

\section{CONSIDERAÇÕES FINAIS}

Depreendeurse que há déficit de conhecimento acerca da pressão arterial e da HAS por parte da população pesquisada, bemcomo urge uma prática da educação emsaúde acerca da HAS mais eficaz e uma maior atenção por parte dos profissionais da saúde quanto à mensuração correta da pressão arterial e à forma de explicar tal fato de modo que se constitua um processo de ensino-aprendizagem

Enbora a aferição da pressão arterial através do método indireto seja umprocedimento simples e fádil, o acesso dos indivíduos aos programas de hipertensão e sua continuidade são bastante dfíceis.

Dessa maneira, é necessário buscar estratégias que permitam maior adesão aos programas e às campanhas de hipertensão, bemcomo às práticas de medidas preventivas. Acreditamos que o início dessa mudança tem como fundamento a atenção primánia voltada para as ações educativas, que pode levar a população, dentro de uma posição áítica, a tomar atitudes que possibilitemsua adaptação a uma condição de vida saudável.

10. FreireP. Eduração deaduttos, hoje Agumas refleões. In Frére P. Políticaeeduração: ensaios. São Paulo(\$P): Cortez, 1993. p. 27.

11 BardnL Arálisedecorteúdb Riodejariero(B): Pescora, 1977.

12. MristéiodaSaúde(BR). Resoluçãon@196de10deautubrode 1996: direnizesenomæs regulametadoras dapesquisaen wolvendb sereshumanos. Braslia(DF); 1996.

13. National nighblood pressureeducation program Theseventh report of the joint national committee on prevention detedion, eveluation, and treaturet of highllood pressure 2003; [ditadb16 abr 2004]. Disponivel emhttp://wwwrhlbi.nihgov/gidelines/ inypertensionlexpress.pdf.

14. Sindtzer SC, BareBG Tratamento depacientes comdistúrbios vesalares coronarianos. In Bnunner \&Suddarth organizadores. Tratado de Enfermagem Médico-Cinúrgico. Rio de Janeiro (B)): GarabaraKoogan; 2002. p. 572-75.

15. Madhado, SC; Sipp, MAC, Leite, JL Cientescomhipertensãoate rid: perspectiva da gerêniadb aidadb. EscAmaNery RevEfferm 2005abr, 9(1): 6471.

16. Fether,CBloodpressuren

17. Sociedade Brasileira de Hipertensão, SociedadeBrasilerra de Cardidogia SociedadeBrasileiradeN Nefrologia Anais da4aDrenizes Brasileiras de Hpettensão; 2002 fev.; Campos dbjordăo( $(P)$, Brasil. SãoPalo: SBHSBCSBN 2002.31p 
Sobre as Autoras

\section{MariaEvidáadeGostro}

Docentelive, Erfermera, Professoradb CursodeEfermagemda UniversicadeEstadul do Ceará enail: evideacastro@baydentecombr

\section{MagaCiviaRolm}

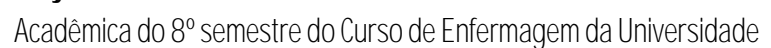
Estad lal do Cerá- LECE; bolsistada Fu ndação CearensedeApoioao Desendimeto tietúficoeTeandógico- PUNCAP. email:falecommaysa@yahoo.combr. 